\title{
Determination and modelling of the particle size dependent residence time distribution in a pilot plant spray dryer
}

\section{Ruprecht, N. ${ }^{\mathbf{a}^{*}}$; Kohlus, R. ${ }^{\mathrm{a}}$}

${ }^{a}$ Department of Process Engineering and Food Powders, University of Hohenheim, Stuttgart, Germany

*E-mail of the corresponding author: nora.ruprecht@uni-hohenheim.de.

\begin{abstract}
The residence time distribution (RTD) in a pilot plant spray dryer was characterised for two kinds of air distributors (centrifugal and parallel flow) and for different atomizing air pressures. To determine the RTD - and the RTD of different particle size fractions - the particle concentration and size at the dryer outlet was measured continuously using a particle counter. Results were modelled using the Bodenstein number and the CSTR in series model. An increasing nozzle pressure leads to a decrease in mean residence time and a more narrow distribution. The influence of nozzle pressure is more pronounced than of air distributor and particle size fraction.
\end{abstract}

Keywords: Residence time distribution; Particle size; Bodenstein number modelling; Nozzle influence; Mechanism of air distribution 


\section{Introduction}

The drying behaviour in a classical spray dryer is determined by the nozzle zone, where the first contact between the hot gas and the product occurs, as well as by the main drying within the drying chamber. In order to describe the drying process, knowledge of the residence time distribution is needed. In addition to the mechanism of air distribution, this is dependent on the nozzle flow and the geometry of the spray dryer. One hypothesis for describing the flow pattern in the drying chamber is based on relatively large vortices leading to different trajectories and residence times depending on the particle inertia or particle size, respectively ${ }^{[1]}$.The residence time of the particles should be sufficient to ensure the desired moisture reduction of the feed but not too long in order to avoid thermal damage like degradation or loss of volatiles. Large deviations in the residence time of different particles, as indicated by a broad residence time distribution (RTD), lead to an inhomogeneous product.

In general, the RTD is determined by tracer injection at the dryer inlet and measuring the response at the outlet by taking samples in predefined time steps and analysing them with respect to their tracer concentration. This tracer can for example be an optically visible ${ }^{[2,3]}$ or conductive ${ }^{[4,5]}$ substance. Especially for fast processes like spray drying this discontinuity of the measurement leads to a too low resolution.

The most common approach for modelling the RTD is using a number of continuous stirred tank reactors (CSTR) in series ${ }^{[6]}$,where the distribution of residence times $\mathrm{E}(\mathrm{t})$ [-] over time $\mathrm{t}[\mathrm{s}]$ is described by the mean residence time $\tau[\mathrm{s}]$ and the number of CSTR in series $\mathrm{N}[-]$.

$$
E(t)=\frac{N}{(N-1) !} \cdot e^{-N \frac{t}{\tau}} \cdot\left(\frac{N t}{\tau}\right)^{N-1}
$$

Various CSTR in series with different tank numbers and residence times can also be set in serial or parallel connection which might account for different flow regimes within the system ${ }^{[4,5]}$.

Another approach for modelling the RTD is the dispersion model, which is based on the Fokker-Planck differential equation, describing the change of concentration c $\left[1 / \mathrm{m}^{3}\right]$ over time $\mathrm{t}[\mathrm{s}]$ and space $\mathrm{z}[\mathrm{m}]$.

$$
\frac{\delta c}{\delta t}=-w \cdot \frac{\delta c}{\delta z}+M_{a x} \cdot \frac{\delta^{2} c}{\delta z^{2}}
$$

$\mathrm{w}[\mathrm{m} / \mathrm{s}]$ is the average particle velocity and $\mathrm{M}_{\mathrm{ax}}\left[\mathrm{m}^{2} / \mathrm{s}\right]$ the axial mixing coefficient describing the derivation from radial flow pattern due to axial dispersion. In dependene of the boundary conditions $\mathrm{M}_{\mathrm{ax}}$ can be calculated directly from the dimensionless variance of the measured RTD. A better way is to fit $\mathrm{M}_{\mathrm{ax}}$ directly to the measured data ${ }^{[7]}$ instead of using these simplifications, although it requires solving the differential equation multiple times. From $\mathrm{M}_{\mathrm{ax}}$ the dimensionless Bodenstein number, which indicates the ratio of convective to dispersive transport, can be calculated. 


$$
B o=\frac{w \cdot L}{M_{a x}}
$$

Here, L [m] is the characteristic length. A low Bodenstein number indicates a broad distribution, a high number a narrow distribution.

Residence time distributions can also be modelled by linking CFD with particle tracing and observing the particle number over time at the outlet ${ }^{[8]}$. Thus, measurements of the RTD can be used to prove theoretical models.

In the present study, the particle RTD was determined using a particle counter. A pulse of maltodextrin was injected into a continuous water stream. When water is sprayed, the droplets evaporate and no particles are recognised by the detector at the dryer outlet. When maltodextrin particles pass the detector, it recognises the particle concentration as well as their size. This set-up allows a continuous measurement of the particle residence time.

\section{Materials and Methods}

\subsection{Experimental set up}

For the spray drying trials a pilot plat spray dryer (GEA Niro, Søborg, Denmark) with the dimensions $3.1 \mathrm{~m} \times 1.2 \mathrm{~m}$ was used. As an injection signal, a $30 \mathrm{~s}$ pulse of $20 \%(\mathrm{w} / \mathrm{w})$ maltodextrin DE 21 (Agrana, Vienna, Austria) solution was injected into a continuous water stream by changing the position of a three-way valve.
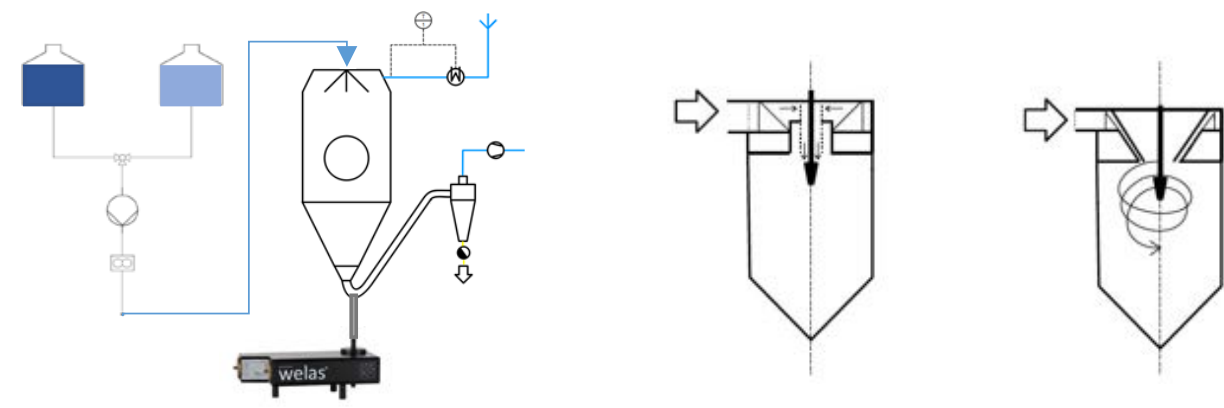

Fig. 1: Experimental set up, parallel and centrifugal air distributor (left to right)

A hollow metal tube with $2 \mathrm{~mm}$ inner diameter, connected to a scattered light detector (Aerolossensor welas 2300, Palas, Karlsruhe, Germany), was inserted in the tower at the outlet of the drying chamber (see Fig. 1). The scattered light detector allows measuring the particle concentration and size within the air stream. From the particle concentration over time the RTD can be calculated.

In order to investigate the effect of the air distributor and nozzle pressure on the RTD the following settings were chosen: 
Table 1: Drying parameters

\begin{tabular}{llll}
\hline Air rate & Feed rate & Inlet temperature air & Outlet temperature air \\
\hline $230 \mathrm{~kg} / \mathrm{h}$ & $9 \mathrm{~kg} / \mathrm{h}$ & $180^{\circ} \mathrm{C}$ & $90^{\circ} \mathrm{C}$ \\
\hline
\end{tabular}

Table 2: Air distributor and nozzle pressure

\begin{tabular}{llcc}
\hline Air distributor & Parallel & \multicolumn{2}{c}{ Centrifugal } \\
Nozzle & 2 fluid nozzle, 2 mm orifice diameter \\
Nozzle pressure (rel.) [bar] & 1 & 2 & 3 \\
Atomizing air rate $[\mathbf{k g} / \mathbf{h}]$ & 12 & 18 & 25 \\
\hline
\end{tabular}

Trials with a nozzle pressure of 1 bar were performed 9 times, with 2 and 3 bar 5 times each.

\subsection{Modelling of the residence time distribution}

The output signal $E_{\text {out }}(t)$, measured by the scattered light detector, results from the convolution of the injection signal $E_{\text {in }}(t)$ with the RTD of the spray dryer.

$$
E_{\text {out }}(t)=E_{\text {in }}(t) * R T D
$$

Here, * indicates the convolution product. Consequently, the RTD can be calculated from deconvolution of the measured output signal with the input signal. Convolution and deconvolution were performed by applying the convolution theorem, whereat numerical Fourier transformation and inverse transformation of discrete data was performed by using the commands implemented in MATLAB ${ }^{\circledR}$. The obtained RTD were fitted to the CSTR in series model (equation 1) and the dispersion model (equation 2) using the method of least squares. The mean residence time $\tau$ was determined from the measured RTD. The mean flow velocity $\mathrm{w}$ for the dispersion model was calculated from $\tau$ and the tower length. Equation 2 was solved numerically using the pdepe solver in MATLAB ${ }^{\circledR}$.

The particle size distribution $\mathrm{q}_{0}[1 / \mathrm{m}]$ for each time step was obtained by the Palas measuring device. The characteristic diameters $\mathrm{d}_{10,0}$ and $\mathrm{d}_{90,0}$ were calculated from the total $\mathrm{q}_{0}$ distribution. For three classes $\left(d<d_{10,0}, d_{10,0}<d<d_{90,0}, d>d_{90,0}\right)$ the residence time distribution was calculated from the number of particles within a class at each time step.

\section{Results and Discussion}

\subsection{Residence time distribution}

With increasing nozzle pressure the measured particle RTD becomes more narrow, as shown in the exemplary curves in Fig. 2. 


\section{Centrifugal}
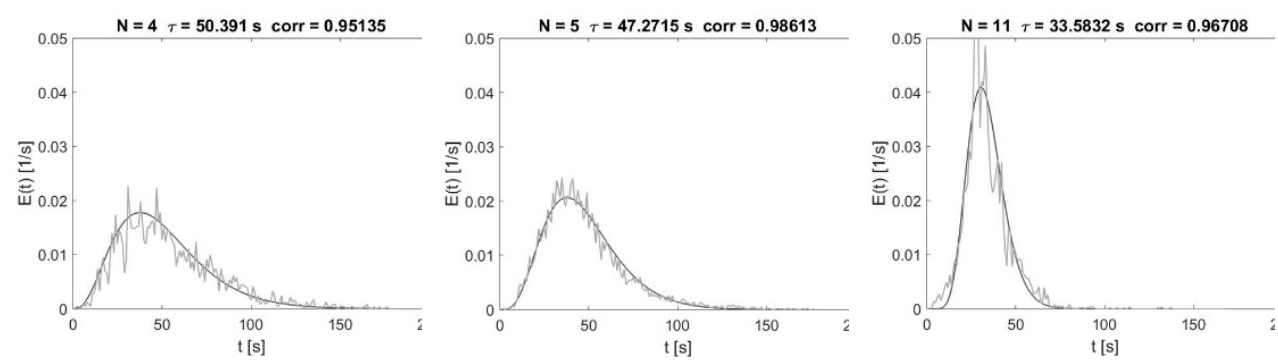

Parallel
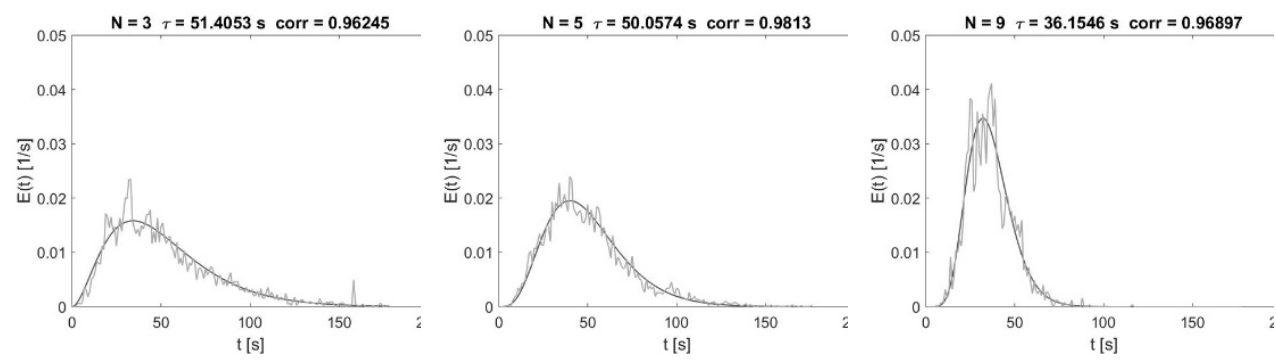

$\Delta \mathrm{p}=1$ bar

$\Delta \mathrm{p}=2$ bar

$\Delta \mathrm{p}=3$ bar

Fig. 2: Influence of nozzle pressure on RTD for centrifugal and parallel air distributor

In the CSTR in series model, this can be seen in an increasing $\mathrm{N}$ (For centrifugal: $\mathrm{N}=4.5-$ 4.4 - 10.8, for parallel $3.4-7.0-8.4$ ). Furthermore there is a decrease in mean residence time $\tau$ with increasing nozzle pressure (For centrifugal: $47.67 \mathrm{~s}-45.84 \mathrm{~s}-35.58 \mathrm{~s}$, for parallel $53.75 \mathrm{~s}-44.48 \mathrm{~s}-37.05 \mathrm{~s}$ ). The latter effect can be seen for both air distributors, whereas no significant difference between the centrifugal $(\bullet)$ and the parallel air distributor ( $\diamond$ can be observed. It was expected that the centrifugal air distributor would lead to an increase in mean residence time.
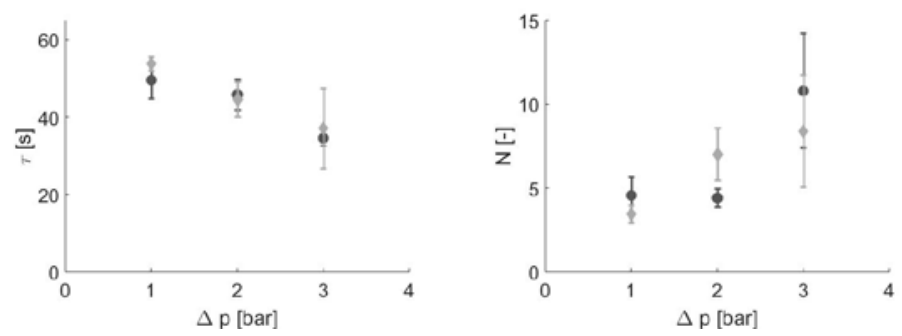

Fig. 3: Mean residence time $\tau$ (left) and number of ideal stirred tank reactors in series $N$ (right) for centrifugal $(\bullet)$ and parallel $(\diamond)$ air distributor at different nozzle pressures 
The mean particle residence time is larger than the mean air residence time, which was calculated as $29 \mathrm{~s}$ at the given process conditions. The flow within a spray chamber can be described by different flow regimes with a fast main central gas jet and a slower flow close to the wall ${ }^{[9]}$. Due to an increasing nozzle pressure there is a higher acceleration of the feed by the pressurized gas and a decrease in spray cone angle. Therefore the amount of particles staying in the faster core jet is increased, which leads to the effects seen in Fig. 2 and Fig. 3. Modelling the flow pattern by using an only one- parametric model is possible, which indicates only little formation of different flow regimes at the tested process conditions.

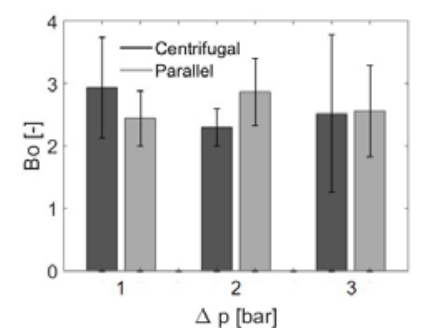

Fig. 4: Bodenstein number for centrifugal and parallel air distributor at different nozzle pressures

The Bodenstein number Bo shows no clear dependency of the nozzle pressure and the air distributor and is approximately constant. The value of the Bodenstein number of 2 - 4 is in accordance with literature data ${ }^{[10]}$.

If the measuring system and injection signal are both constant, variations in the RTD are due to variations in the process, e.g. spontaneous formation of vortices. The RTDs, fitted to the CSTR in series model, are shown in Fig. 5 for all 9 trials at 1 bar nozzle pressure.
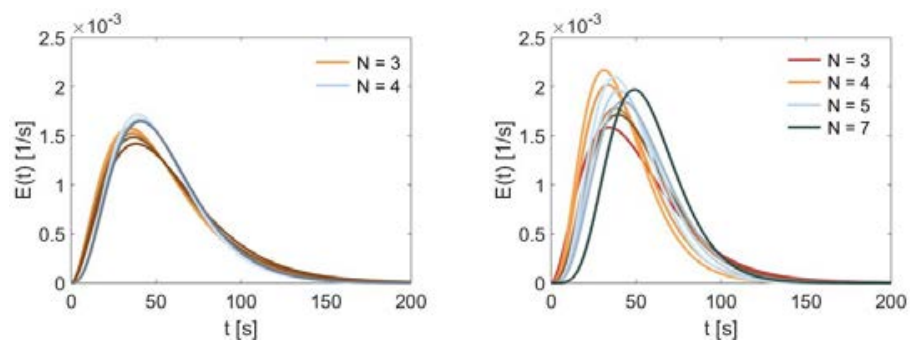

Fig. 5: Variations in RTD for parallel (left) and centrifugal (right) air distributor at 1 bar nozzle pressure.

The RTDs measured with the parallel air distributor can be modelled by using $\mathrm{N}=3$ and $\mathrm{N}=4$. The mean residence time $\tau$ is in the range of $51.4 \mathrm{~s}$ to $57.4 \mathrm{~s}$. The RTDs measured with the centrifugal air distributor show a larger variance with a $\mathrm{N}$ of 3, 4, 5 and 7 and $\tau$ from 41.4 $\mathrm{s}$ to $57.1 \mathrm{~s}$. The flow within the spray drying chamber caused by the centrifugal air distributor seems to be less stable and more susceptible to fluctuations as indicated by this larger variance. 


\subsection{Particle size dependent residence time distribution}

In Fig. 6 the RTDs from Fig. 2 are shown for different size fractions.

Centrifugal
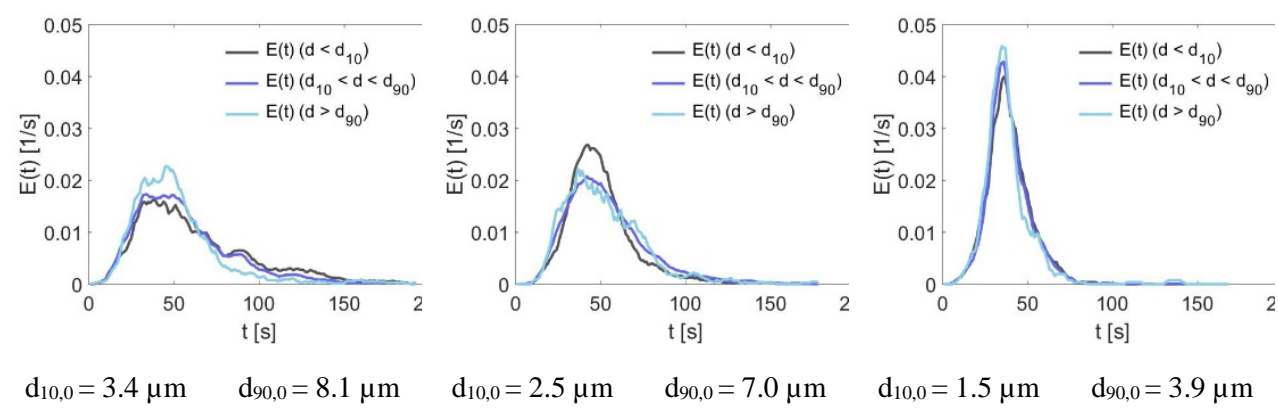

Parallel
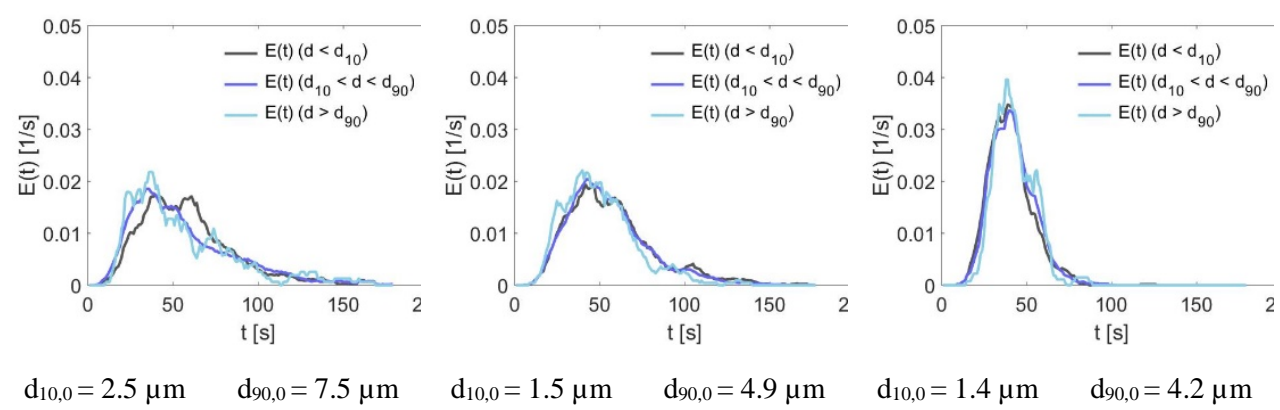

$$
\mathrm{d}_{10,0}=2.5 \mu \mathrm{m} \quad \mathrm{d}_{90,0}=7.5 \mu \mathrm{m}
$$

$\Delta \mathrm{p}=1$ bar

$\Delta \mathrm{p}=2$ bar

$\Delta \mathrm{p}=3$ bar

Fig. 6: Influence of nozzle pressure on RTD for different particle size fractions for centrifugal and parallel air distributor

The mean particle size decreases with increasing nozzle pressure due to the reduction of initial droplet size. No significant difference between the RTDs of the different size fractions can be observed. Thus, the effects of an increasing nozzle pressure on RTD (see Fig. 3) are not caused by the decreased particle size.

It was expected that different particle sizes will lead to different trajectories and therefore to different residence times ${ }^{[1]}$. As indicated by the small difference in between $d_{10,0}$ and $d_{90,0}$, the particle size distribution is narrow which might lead to no size fraction effects in the RTD being visible. 


\section{Conclusions}

The residence time distribution in a pilot plant spray dryer was characterised for different kinds of air distributors (centrifugal and parallel flow) as well as for different atomizing air pressures (1, 2 and 3 bar relative pressure). The measurement is based on a quasi-continuous acquisition of the particle concentration and size at the dryer outlet using a scattered light detector. The measured RTDs can be described by models with only one parameter, like the CSTR in series model, which indicates only little formation of different flow regimes at the tested process conditions.

An increasing nozzle pressure leads to a decrease in mean residence time and a more narrow distribution but the mean particle residence time is larger than the mean air residence time. The influence of nozzle pressure is more pronounced than of air distributor and particle size fraction.

In further studies the effect of a broader particle size distribution will be investigated.

\section{References}

[1] Rähse, W.; Dicoi, O. Produktdesign disperser Stoffe: Industrielle Sprühtrocknung. Chemie Ingenieur Technik 2009, 81(6), 699-716.

[2] Kieviet, F.; Kerkhof, P.J. Measurements of Particle Residence Time Distributions in A Co-Current Spray Dryer. Drying Technology 1995, 13(5-7), 1241-1248.

[3] Bachmann, P.; Bück, A.; Tsotsas, E. Investigation of the residence time behavior of particulate products and correlation for the Bodenstein number in horizontal fluidized beds. Powder Technology 2016, 301, 1067-1076.

[4] Tylor, T. Powder and Air Residence Time Distributions in Countercurrent Spray Dryers. Proceedings of 9th International Drying Symposium 1994, Vol. A, 463-470.

[5] Jeantet, R.; Ducept, F.; Dolivet, A.; Méjean, S.; Schuck, P. Residence time distribution: A tool to improve spray-drying control. Dairy Science and Technology 2008, 88(1), 31-43.

[6] Levenspiel, O. Chemical reaction engineering, 2nd ed; Wiley: New York, 1972.

[7] Bachmann, P.; Tsotsas, E. Analysis of Residence Time Distribution Data in Horizontal Fluidized Beds. Procedia Engineering 2015, 102, 790-798.

[8] Kieviet, F.G. Modelling quality in spray drying, 1997.

[9] Blei, S.; Sommerfeld, M. CFD in Drying Technology- Spray-Dryer Simulation. In Modern Drying Technology: Comutational Tools at Different Scales; Tsotsas, E., Mujumbar, A.S., Eds.; Wiley, 2007; 155-204.

[10] Fitzer, E.; Fritz, W. Technische Chemie: Eine Einführung in die chemische Reaktionstechnik ; mit 36 Tabellen und 31 Rechenbeispielen, 2nd ed; Springer: Berlin, 1982. 